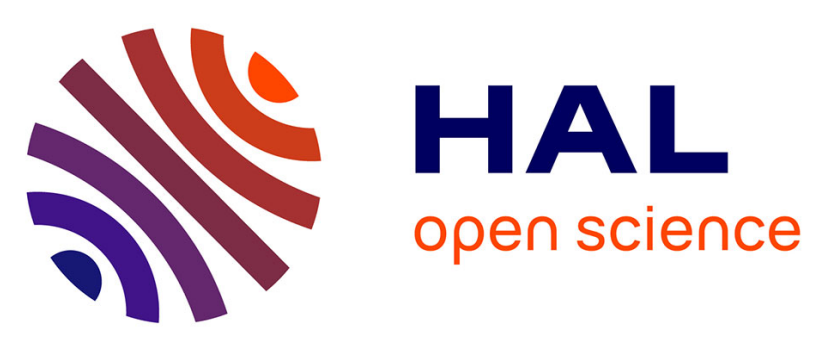

\title{
On a simple cyclic plasticity modeling with implicit kinematic hardening restoration
}

Boumediene Nedjar, Dang Truc Nguyen

\section{To cite this version:}

Boumediene Nedjar, Dang Truc Nguyen. On a simple cyclic plasticity modeling with implicit kinematic hardening restoration. Annals of Solid and Structural Mechanics, 2012, 4 (1-2), pp.33-42. 10.1007/s12356-012-0031-2 . hal-00782039

\section{HAL Id: hal-00782039 \\ https://hal-enpc.archives-ouvertes.fr/hal-00782039}

Submitted on 30 Jan 2013

HAL is a multi-disciplinary open access archive for the deposit and dissemination of scientific research documents, whether they are published or not. The documents may come from teaching and research institutions in France or abroad, or from public or private research centers.
L'archive ouverte pluridisciplinaire HAL, est destinée au dépôt et à la diffusion de documents scientifiques de niveau recherche, publiés ou non, émanant des établissements d'enseignement et de recherche français ou étrangers, des laboratoires publics ou privés. 


\title{
On a simple cyclic plasticity modeling with implicit kinematic hardening restoration
}

\author{
B. Nedjar · D.T. Nguyen
}

Received: date / Accepted: date

\begin{abstract}
This paper presents a fully three-dimensional plastic constitutive modeling framework suitable for the prediction of cyclic loading at large number of cycles. It can require only one yield surface and it is motivated by a simple rheological model where a restoration of the kinematic hardening is introduced. The classical kinematic hardening rules are then simply adapted leading to time-dependent evolution laws that are consistent with continuum thermodynamics requirements. The resulting behavior is physically motivated by many man-made materials of engineering interest such as bituminous material. This framework allows all types of yield functions to be easily implemented numerically. This is first illustrated with algorithmic details through a simple associative pressure-insensitive model example of the von Mises type. Then a more elaborated model is given where the present framework is applied to the description of bituminous materials submitted to triaxial static creep and to large number of cyclic loadings. Of particular interest is the ratcheting and the mean stress relaxation. The responses agree well with some experimental test results found in the literature.
\end{abstract}

Keywords Kinematic hardening restoration · Viscoplasticity · Continuum thermodynamics $\cdot$ Large number cylic loading $\cdot$ Numerical implementation

D.T. Nguyen

Université Paris-Est, Laboratoire Navier (UMR 8205), CNRS, ENPC, IFSTTAR

F-77455 Marne-la-Vallée, France

E-mail: Truc.Nguyen@scyna4.fr

B. Nedjar

Université Paris-Est, Laboratoire Navier (UMR 8205), CNRS, ENPC, IFSTTAR

F-77455 Marne-la-Vallée, France

Tel. +33-1-64153715

E-mail: boumediene.nedjar@enpc.fr 


\section{Introduction}

Plastic accumulation is one of the major distresses of materials and structures under cyclic loadings. This phenomenon designates in general the apparition and accumulation of permanent deformations with the increasing number of loading cycles. For many materials, this accumulation may not cease until fracture. In the particular domain of pavement engineering, the ratcheting of asphalt concrete is the origin of the rutting phenomenon, an important distress mode under repeated traffic loading.

During the last years, many efforts have been devoted to model the ratcheting responses of various materials. These works concentrated especially on metal-like materials, see for example $[7,11,12,20]$ among many others. In general, these models postulate single surface elastic domain with complex nonlinear kinematic and isotropic hardening rules and have proven their relevance on the modeling of such metal-like materials. On the other hand, the multimechanisms and multi-criteria type models, generalized by Mandel in [8], are also able to reproduce complex phenomena under cyclic loadings. Here again, the relevance of these approaches has been clearly demonstrated, for instance in $[2,6,10]$ to mention a few. However, the application of multi-surface models to complex problems is not obvious, mostly because of the numerical difficulties due to singular points when multiple pressure-sensitive yield criteria are used. This was experienced for instance by the authors in $[9,10]$. Note that, in general, the use of pressure-sensitive yield criteria is necessary when modeling geomaterials, concretes or bituminous materials. In particular for these latters, advances have been reported by a number of authors, see for example [14-16, 19] among others. The relevant long literature has contributed significantly to the understanding of the mechanisms influencing the asphalt concrete performance as well, and most of the proposed models are more adapted to the predictive material responses under monotonic loadings. A model that is able to predict at the same time the material responses under monotonic and large number cyclic loadings is still a challenging task.

The constitutive modeling framework proposed in this paper is precisely motivated by physical observations on bituminous materials. In such mixtures, the permanent deformations occur as a result of slight movements between the aggregates at the meso-scale. These movements are accompanied by the viscous flow of the asphalt binder. As a consequence, the material response is viscoplastic at the macroscopic level. Moreover, it is also observed that the viscous flow of the binder relaxes the auto-stresses due to the incompatibility of the plastic deformations. This relaxation phenomenon constitutes the point of departure of the present formulation resulting in the so-called concept of kinematic hardening restoration. That is, the kinematic hardening stresses (the back-stresses in the continuum thermodynamics terminology) still relax even when the plastic straining ceases. The relaxation process developed in this work is based on a differential evolution equation not resorting to history variables, as that was the case in earlier models found in the literature. In fact, the concept of kinematic hardening restoration is not new. It has already been 
used for metals, precisely, by using history variable models, see for instance [7]. The framework we develop in this paper may be applied not only to bituminous materials, but also to other materials such as polymers and even for metals as well.

An outline of the remainder of the paper is as follows. In section 2 , the formulation is firstly motivated by a simple rheological model where the governing equations are derived by elementary considerations. Of particular interest, it is shown that this model is able to exhibit plastic accumulation under both static and cyclic loadings. A straightforward three-dimensional generalization is given in Section 3 following the basic concepts of the continuum thermodynamics. Then, in section 4 , a simple $J_{2}$ flow-like model example of the von Mises type is detailed from the theoretical and numerical points of view to show how plastic accumulations at large number of cycles can be simply implemented by standard numerical schemes. In section 5, a more elaborated model is briefly presented. This latter is adapted to bituminous materials and a set of numerical results are compared with some experimental responses of [1]. Finally, conclusions and perspectives are drawn in section 6. Noteworthy remarks and comments are given throughout this paper.

\section{Motivation: a one-dimensional rheological model}

We first illustrate the basic idea behind the present modeling framework through a one-dimensional study. For this, let us examine the different rheological models depicted in Figure 1.

- The classical Prager model of Figure 1(a) is the simplest one that is capable to produce time-independent permanent strains with kinematic hardening. It consists of a spring of constant $E>0$ in series with a plastic device composed by a friction element with constant $\sigma_{y}>0$ in parallel with a spring of constant $H>0$ (the kinematic hardening modulus in our case).

- In Figure 1(b), a parallel dashpot of constant $\eta>0$ is added to the last plastic device. The resulting model is then no more than an extension of the Prager model that renders the plastic evolution time-dependent.

- Finally, in the model depicted in Figure 1(c), we simply replace the last spring of constant $H$ by a Maxwell element. That is, the stress in the spring of constant $H$ is relaxed by a dashpot of constant $\eta_{X}>0$. Notice that this replacement can also be done on the Prager model of Figure 1(a), i.e. without the dashpot of constant $\eta$. In all cases, the global constitutive behavior will always be time-dependent by construction.

Now the governing equations for the rheological model of Figure 1(c) are derived by completely elementary considerations. Let $\sigma$ denotes the total stress acting on it, $\varepsilon$ the total strain, and $\varepsilon^{\mathrm{p}}$ the plastic strain. Moreover, let $\alpha$ be the strain in the spring of constant $H$, and $\beta$ the strain in the dashpot of constant $\eta_{X}$. One has then:

$$
\sigma=E\left(\varepsilon-\varepsilon^{\mathrm{p}}\right), \quad X=H \alpha, \quad \varepsilon^{\mathrm{p}}=\alpha+\beta
$$




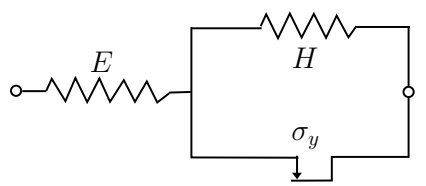

(a)

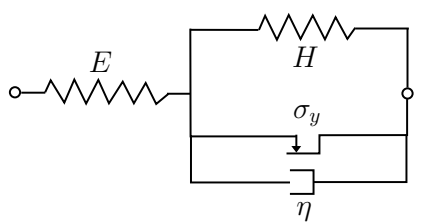

$(b)$

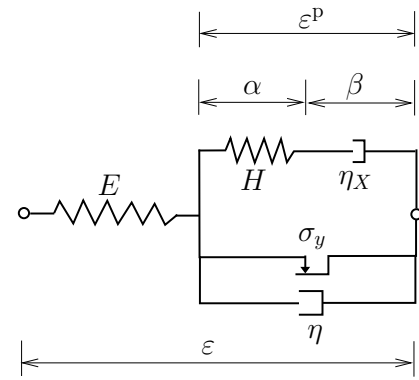

$(c)$

Fig. 1 One-dimensional rheological models: (a) the Prager model, (b) the Bingham model, (c) the model for the present formulation.

where the (back-)stress $X$ represents the kinematic hardening. The elastic domain $f$, represented by the friction element of constant $\sigma_{y}$, is here written as $f=|\sigma-X|-\sigma_{y}$, and the plastic flow is given by:

$$
\dot{\varepsilon}^{\mathrm{p}}=\gamma \frac{\partial f}{\partial \sigma}
$$

where the scalar $\gamma$ can be given by a viscoplastic regularization equation of the Perzyna type [13]:

$$
\gamma=\frac{\langle f\rangle}{\eta}
$$

where $\langle$.$\rangle defines the positive part function as \langle f\rangle=\frac{1}{2}(f+|f|)$. Now, assuming a linear relation connecting the stress $X$ with its corresponding strain rates $\dot{\beta}$, i.e. $X=\eta_{X} \dot{\beta}$, the evolution of the strain-like variable $\alpha$ is obtained from the rate form of Equation. $(1)_{3}$ :

$$
\dot{\alpha}=\dot{\varepsilon}^{\mathrm{p}}-\frac{X}{\eta_{X}}
$$

Analyzing the above evolution equations, it follows that for situations where loadings are such that $f \leq 0$, Equation (3) gives $\gamma=0$ leading to $\dot{\varepsilon}^{\mathrm{p}}=0$ by Equation (2), and Equation (4) becomes then:

$$
\dot{\alpha}=-\frac{X}{\eta_{X}}
$$


That is, the kinematic hardening continues to evolve even when the stress state belongs to the interior of the elastic domain, i.e. with $f \leq 0$. In this case, the back-stress $X$ is restored through the following evolution equation obtained by combining Equation (5) with rate form of the constitutive relation $(1)_{2}$ :

$$
\dot{X}+\frac{H}{\eta_{X}} X=0
$$

Remark 1. If the dashpot of constant $\eta$ is removed from the rheological model of Figure 1(c), the scalar $\gamma$ in Equation (2) becomes a plastic multiplier determined by the classical Kuhn-Tucker loading/unloading conditions: $\gamma \geq 0$, $f \leq 0$ and $\gamma f=0$, together with the consistency condition $\gamma \dot{f}=0$. In this case the evolution equations (4)-(6) remain the same.

As an illustration, Figure 2 shows the results of a static and a cyclic creep semi-analytical computations obtained with the constitutive equations (1) (4). The used material parameters are: $E=3000 M P a, \sigma_{y}=0.1 M P a, H=$ $80 \mathrm{MPa}, \eta=2500 \mathrm{MPa}$ s and $\eta_{X}=10^{6} \mathrm{MPa}$.

For the static creep of Figure 2(a), a stress of value $\sigma=0.25 \mathrm{MPa}$ has been quickly applied at time $t=0$ and then maintained. One can observe that the plastic strain $\varepsilon^{\mathrm{p}}$ continue growing with time. And in Figure 2(b), a sinusoidal loading has been applied with an amplitude of $\sigma_{\max }=0.25 \mathrm{MPa}$ at a frequency of $1 \mathrm{~Hz}$. The evolution of the plastic strain $\varepsilon^{\mathrm{p}}$ is plotted versus the number of cycles. One can observe that the plastic accumulation grows contiuously even at very large number of cycles.

Hence, both the static and cyclic creep are simultaneously described by the same rheological model where the kinematic hardening restoration is in turn controlled by the viscous parameter $\eta_{X}$ through the evolution equation (4).

\section{Three-dimensional generalization and continuum thermodynamics}

The extension of the precedent one-dimensional developments to the threedimensional constitutive modeling is straightforward. As usual in the infinitesimal theory, the total strain tensor $\varepsilon$ is additively split into an elastic part $\varepsilon^{\mathrm{e}}$ and a plastic part $\varepsilon^{\mathrm{p}}: \varepsilon=\varepsilon^{\mathrm{e}}+\varepsilon^{\mathrm{p}}$. From the continuum thermodynamics point of view, the state of the material is characterized by its free energy function denoted here by $\psi$. In our context, this latter is chosen of the form $\psi \equiv \psi\left(\varepsilon^{\mathrm{e}}=\boldsymbol{\varepsilon}-\varepsilon^{\mathrm{p}}, \boldsymbol{\alpha}\right)$ where the tensor quantity $\boldsymbol{\alpha}$ is the strain-like internal variable related to kinematic hardening. Without loss of generality, isotropic hardening will not be considered for the sake of clarity. This latter can in fact be included in the formulation in a usual manner.

In the simplest case, the elastic domain $\mathbb{E}$ is defined by a single-surface yield criterion of the form

$$
\mathbb{E}=\{(\boldsymbol{\sigma}, \boldsymbol{X}) / f(\boldsymbol{\sigma}, \boldsymbol{X}) \leq 0\}
$$




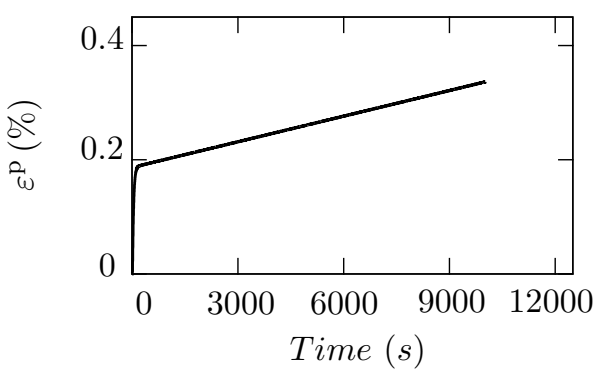

$(a)$

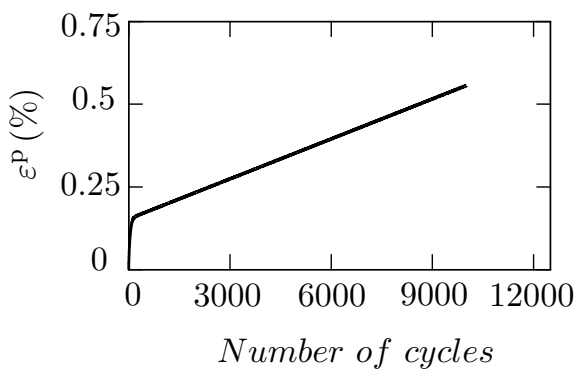

$(b)$

Fig. 2 Responses of the rheological model of Figure 1(c): (a) static creep, (b) cyclic creep.

where $f$ is a convex function, $\boldsymbol{\sigma}$ is the stress tensor, and $\boldsymbol{X}$ denotes the stresslike internal variable conjugated to $\boldsymbol{\alpha}$ in the sense that

$$
\boldsymbol{X}=\frac{\partial \psi}{\partial \boldsymbol{\alpha}}
$$

this latter being in fact the set of back-stress tensors. By taking the time derivative of $\psi$, inserting the result into the Clausius-Duhem inequality, i.e. $\mathcal{D}=\boldsymbol{\sigma}: \dot{\varepsilon}-\dot{\psi} \geq 0$, and making use of standard arguments in continuum thermodynamics [5], one obtains the classical state law

$$
\boldsymbol{\sigma}=\frac{\partial \psi}{\partial \varepsilon^{\mathrm{e}}}
$$

together with the reduced dissipation inequality

$$
\mathcal{D}=\boldsymbol{\sigma}: \dot{\varepsilon}^{\mathrm{p}}-\boldsymbol{X}: \dot{\boldsymbol{\alpha}} \geq 0
$$

where the symbol : denotes the double contraction, i.e. $\mathbf{A}: \mathbf{B}=\operatorname{tr}\left[\mathbf{A B}^{T}\right] \equiv$ $A_{i j} B_{i j}$ (summation on repeated indices is assumed), and $\operatorname{tr}[$.$] designates the$ trace operator.

Now motivated by the rheological model of Figure 1(c), the plastic strain rate is in turn additively split in the form $\dot{\boldsymbol{\varepsilon}}^{\mathrm{p}}=\dot{\boldsymbol{\alpha}}+\dot{\boldsymbol{\beta}}$ where $\dot{\boldsymbol{\beta}}$ is the viscous kinematic hardening strain rate tensor. The inequality (10) becomes then:

$$
\mathcal{D}=(\boldsymbol{\sigma}-\boldsymbol{X}): \dot{\boldsymbol{\varepsilon}}^{\mathrm{p}}+\boldsymbol{X}: \dot{\boldsymbol{\beta}} \geq 0
$$


In view of the expression of the first term in (11), the yield surface defining the elastic domain in (7) is restricted to the general form $f \equiv f(\boldsymbol{\sigma}-\boldsymbol{X})$. For the kinematic hardening restoration, we assume the existence of a potential denoted by $\Omega_{\mathrm{r}}(\boldsymbol{X})$, i.e. a convex and positive function such that $\Omega_{\mathrm{r}}=0 \Leftrightarrow$ $\boldsymbol{X}=\mathbf{0}$. Then, the evolution equations can be given by

$$
\left\{\begin{array}{l}
\dot{\varepsilon}^{\mathrm{p}}=\gamma \frac{\partial f}{\partial(\boldsymbol{\sigma}-\boldsymbol{X})} \equiv \gamma \frac{\partial f}{\partial \boldsymbol{\sigma}} \\
\dot{\boldsymbol{\beta}}=\frac{\partial \Omega_{\mathrm{r}}}{\partial \boldsymbol{X}} \quad \Longrightarrow \dot{\boldsymbol{\alpha}}=\dot{\boldsymbol{\varepsilon}}^{\mathrm{p}}-\frac{\partial \Omega_{\mathrm{r}}}{\partial \boldsymbol{X}}
\end{array}\right.
$$

With this flow rule, the thermodynamic requirement (11) is satisfied for any admissible process thanks to the convexity of the functions $f$ and $\Omega_{\mathrm{r}}$ together with the positiveness of the multiplier $\gamma$. The evolution equation $(12)_{1}$ is the three-dimensional extension of its one-dimensional counterpart given in (2). Likewise, with a quadratic potential $\Omega_{\mathrm{r}}=\boldsymbol{X}: \boldsymbol{X} / 2 \eta_{X},(12)_{2}$ extends (4) to three-dimensions.

\section{$4 \mathrm{~A} J_{2}$-flow model example}

To make matters as concrete as possible, we consider in detail a simple plastic model of the von Mises type with a pressure insensitive yield criterion. We denote by $s$ and $\boldsymbol{e}$ the deviatoric stress and strain tensors, respectively

$$
s=\boldsymbol{\sigma}-p \mathbf{1}, \quad e=\varepsilon-\frac{1}{3} \operatorname{tr}[\varepsilon] \mathbf{1}
$$

where 1 denotes the second order unit tensor and $p=\frac{1}{3} \operatorname{tr}[\boldsymbol{\sigma}]$ the hydrostatic stress. Considering again only kinematic plastic hardening, the yield criterion is chosen as

$$
f(\boldsymbol{\sigma}, \boldsymbol{X})=\|\boldsymbol{s}-\boldsymbol{X}\|-\sqrt{\frac{2}{3}} \sigma_{y}
$$

with $\sigma_{y}$ being the flow stress and where the norm of a second order tensor is defined as $\|\|=.[(.):(.)]^{1 / 2}$. By construction, the back-stress tensor is purely deviatoric, i.e. $\operatorname{tr}[\boldsymbol{X}]=0$. The constitutive relations $(8)-(9)$ are given by

$$
\boldsymbol{\sigma}=\boldsymbol{C}:\left(\varepsilon-\varepsilon^{\mathrm{p}}\right), \quad \boldsymbol{X}=H \boldsymbol{\alpha}
$$

where $\boldsymbol{C}$ is the rank four Hooke's elasticity tensor ( $\lambda$ and $\mu$ being the Lamé coefficients), and the scalar $H$ is the plastic kinematic hardening modulus considered constant in this example. In other words, the free energy function $\psi$ is given by the simple quadratic form

$$
\psi=\frac{1}{2} \varepsilon^{\mathrm{e}}: \boldsymbol{C}: \varepsilon^{\mathrm{e}}+\frac{1}{2} H \boldsymbol{\alpha}: \boldsymbol{\alpha}
$$


The model is completed by appending the evolution equations of the form given by (12) for the internal variables:

$$
\begin{aligned}
& \dot{\boldsymbol{\varepsilon}}^{\mathrm{p}}=\gamma \underbrace{\frac{\boldsymbol{s}-\boldsymbol{X}}{\|\boldsymbol{s}-\boldsymbol{X}\|}}_{=\hat{\boldsymbol{n}}} \\
& \dot{\boldsymbol{\alpha}}=\gamma \hat{\boldsymbol{n}}-\frac{\boldsymbol{X}}{\eta_{X}}
\end{aligned}
$$

where $\hat{\boldsymbol{n}}$ is the unit normal to the yield surface, and where again the potential $\Omega_{\mathrm{r}}(\boldsymbol{X})=\boldsymbol{X}: \boldsymbol{X} / 2 \eta_{X}$ has been used. In view of the numerical resolution, instead to deal with the evolution equation $(17)_{2}$, we prefer its equivalent conjugate form by use of the rate form of the constitutive relation $(15)_{2}$ :

$$
\dot{\boldsymbol{X}}=\gamma H \hat{\boldsymbol{n}}-\frac{H}{\eta_{X}} \boldsymbol{X}
$$

\subsection{Numerical approximation}

The algorithmic approximation of the local evolution equations $(17)_{1}$ and (18) is based on the nowadays well-known elastic predictor/plastic corrector scheme. From the finite element point of view, this procedure is accomplished locally at each integration point.

Let $\left[t_{n}, t_{n+1}\right]$ be a typical time interval. For an arbitrary material point, the known (internal) variables $\left\{\varepsilon_{n}^{\mathrm{p}}, \boldsymbol{X}_{n}\right\}$ are considered as prescribed initial data. Then, within the elastic predictor step, the trial state is obtained by freezing the plastic flow ignoring the constraint placed by the plastic yield criterion. The trial state is obtained by mere function evaluation as

$$
\boldsymbol{s}_{n+1}^{\text {trial }}=\boldsymbol{s}_{n}+2 \mu \Delta \boldsymbol{e}_{n+1}, \quad p_{n+1}^{\text {trial }}=p_{n}+\kappa \Delta \boldsymbol{\varepsilon}_{n+1}: \mathbf{1}
$$

where $\kappa=\lambda+\frac{2}{3} \mu$ is the bulk modulus, $\Delta \varepsilon_{n+1}$ is the total strain increment and $\Delta \boldsymbol{e}_{n+1}$ its deviatoric part. The yield criterion is then evaluated as

$$
f_{n+1}^{\text {trial }}=\left\|\boldsymbol{s}_{n+1}^{\text {trial }}-\boldsymbol{X}_{n}\right\|-\sqrt{\frac{2}{3}} \sigma_{y}
$$

- On the one hand, if $f_{n+1}^{\text {trial }}>0$, then $\gamma>0$ and an incrementally plastic straining process is taking place. A plastic correction has to be performed to get the final solution as

$$
\left\{\begin{array}{l}
\boldsymbol{s}_{n+1}=\boldsymbol{s}_{n+1}^{\mathrm{trial}}-2 \mu \Delta \gamma \hat{\boldsymbol{n}}_{n+1} \\
\boldsymbol{X}_{n+1}=\boldsymbol{X}_{n}+H \Delta \gamma \hat{\boldsymbol{n}}_{n+1}-\frac{H}{\eta_{X}} \Delta t \boldsymbol{X}_{n+1}
\end{array}\right.
$$


where $\Delta \gamma=\gamma \Delta t$ with $\Delta t=t_{n+1}-t_{n}$, and where an implicit backward Euler finite difference scheme has been employed for the evolution equations $(17)_{1}$ and (18). Equation $(21)_{2}$ can be conveniently rewritten as

$$
\frac{\boldsymbol{X}_{n+1}}{\chi}=\boldsymbol{X}_{n}+H \Delta \gamma \hat{\boldsymbol{n}}_{n+1} \quad \text { with } \chi=\frac{\eta_{X}}{\eta_{X}+H \Delta t}
$$

Here the tensor $\hat{\boldsymbol{n}}_{n+1}$ at the final state has the remarkable property that it is also given by the known trial state as

$$
\hat{\boldsymbol{n}}_{n+1}=\frac{\boldsymbol{s}_{n+1}-\boldsymbol{X}_{n+1}}{\left\|\boldsymbol{s}_{n+1}-\boldsymbol{X}_{n+1}\right\|} \equiv \frac{\boldsymbol{s}_{n+1}^{\mathrm{trial}}-\chi \boldsymbol{X}_{n}}{\left\|\boldsymbol{s}_{n+1}^{\mathrm{trial}}-\chi \boldsymbol{X}_{n}\right\|}
$$

Finally, the unknown multiplier $\Delta \gamma$ is obtained by enforcing the consistency condition written as $f_{n+1}=(\eta / \Delta t) \Delta \gamma$ to avoid numerical difficulties when $\eta \rightarrow 0$, see for exemple [17]. It is explicitely given by

$$
\Delta \gamma=\frac{\left\|\boldsymbol{s}_{n+1}^{\text {trial }}-\chi \boldsymbol{X}_{n}\right\|-\sqrt{\frac{2}{3}} \sigma_{y}}{2 \mu+\chi H+\frac{\eta}{\Delta t}}
$$

where the relation $\left\|\boldsymbol{s}_{n+1}-\boldsymbol{X}_{n+1}\right\|=\left\|\boldsymbol{s}_{n+1}^{\text {trial }}-\chi \boldsymbol{X}_{n}\right\|-\Delta \gamma(2 \mu+\chi H)$ has been used.

- On the other hand, if $f_{n+1}^{\text {trial }} \leq 0$, then $\gamma=0$ and the trial state is the final solution with also $\varepsilon_{n+1}^{\mathrm{p}}=\varepsilon_{n}^{\mathrm{p}}$, except for the back-stress tensor where the kinematic hardening restoration takes place through the following evolution equation, see Equation (18):

$$
\dot{\boldsymbol{X}}=-\frac{H}{\eta_{X}} \boldsymbol{X}, \quad \text { with }\left.\boldsymbol{X}\right|_{t=t_{n}}=\boldsymbol{X}_{n}
$$

This latter can be approximated numerically by an exponential scheme as

$$
\boldsymbol{X}_{n+1}=\boldsymbol{X}_{n} \exp \left[-\frac{H}{\eta_{X}} \Delta t\right] .
$$

For the sake of clarity, Table 1 summarizes the conceptual steps involved during this local resolution procedure.

\subsection{Representative numerical examples}

As an illustration, we consider a $(5 \times 5 \times 10) \mathrm{mm}^{3}$ parallelepipedic sample discretized with $(4 \times 4 \times 4)$ classical 8 -nodes hexahedral elements. The specimen is firstly submitted to a cyclic uniform pressure on the top face with an amplitude of $200 \mathrm{KPa}$ at a period $T=40 \mathrm{~s}$. The rest of the boundary conditions are such that the bottom face is fixed vertically (along the $\mathbf{e}_{3}$ direction) while leaving the displacements free on its plane, and the lateral faces of the sample are completely free. Figure 3 shows the resulting stress-strain curves 
Table 1 Local algorithm for the plastic evolution.

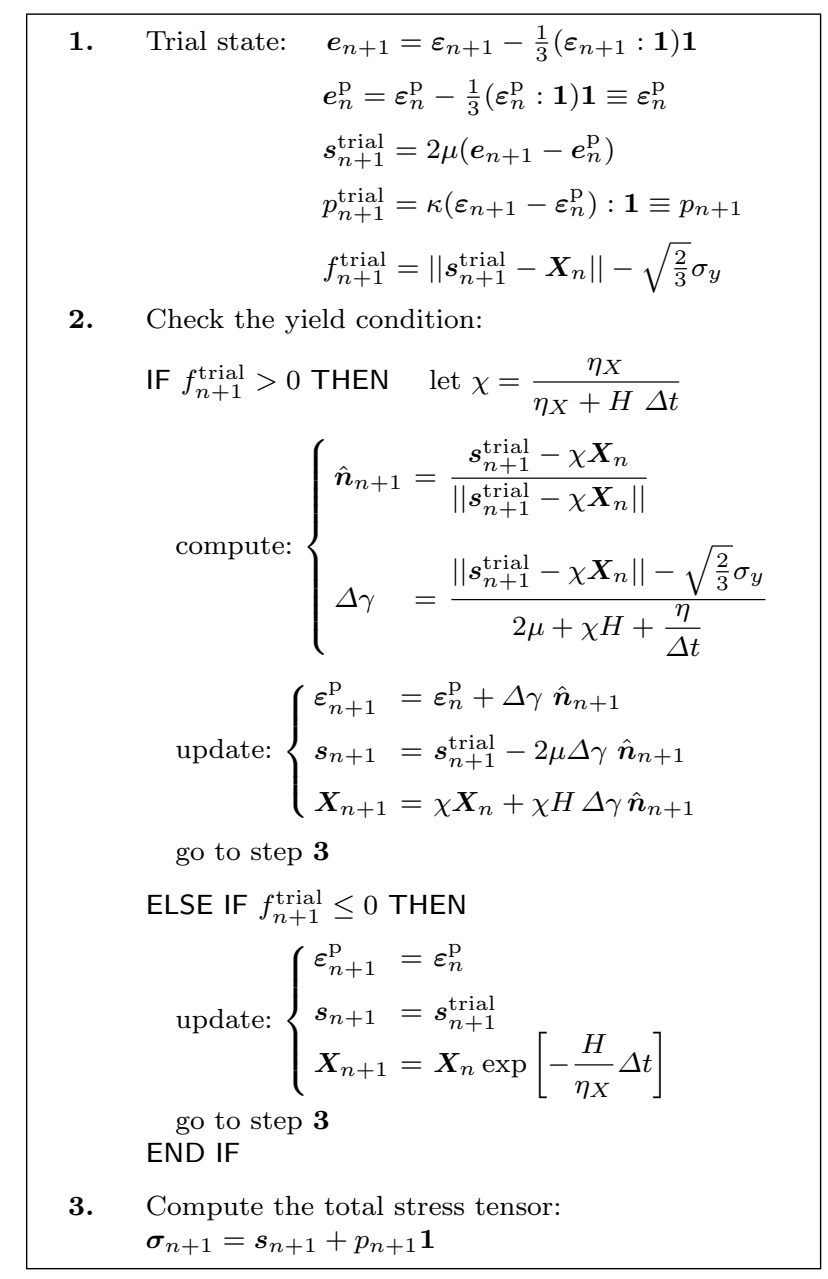

(in the $\mathbf{e}_{3}$ direction) of two different computations: the first one with $\eta=0$ and the second one with $\eta=750 \mathrm{MPas}$. The common material parameters used for both the computations are: $E=7500 \mathrm{MPa}, \nu=0.3, \sigma_{y}=0.06 \mathrm{MPa}$, $H=250 \mathrm{MPa}$ and $\eta_{X}=4510^{3} \mathrm{MPas}$.

Notice that even for $\eta=0$, the material response is still time-dependent through the kinematic hardening restoration process (see also the comments of Remark 1). Irrespective to the value of the parameter $\eta$, plastic accumulation grows with the number of cycles.

To show again the ability of the present model for more complex loading conditions, a non-homogeneous cyclic loading is this time simulated on the same sample. Here a pressure of amplitude $400 \mathrm{KPa}$ at a period $\mathrm{T}=40 \mathrm{~s}$ is applied on one fourth of the top face as shown in Figure 4. The same boundary 


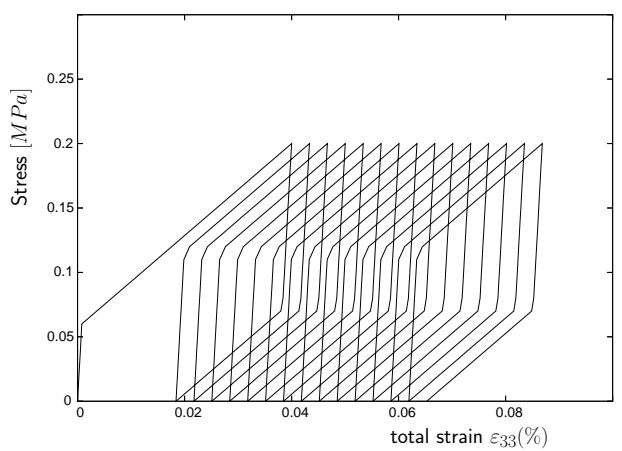

(a)

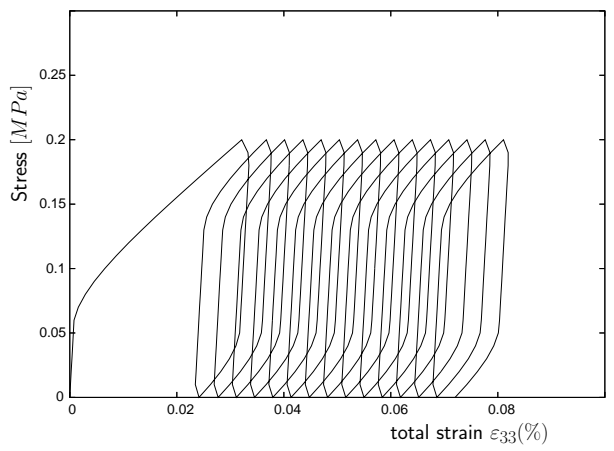

Fig. 3 Homogeneous cyclic loading of amplitude $200 \mathrm{KPa}$ and period $\mathrm{T}=40 \mathrm{~s}$ with: (a) $\eta=0$, and (b) $\eta=750 \mathrm{MPas}$.

conditions on the other faces and the same material parameters as for the precedent computations are used with $\eta=750 \mathrm{MPas}$. Figure 4 depicts the evolution of resultant force versus the maximum displacement of the upper right nodes.

Remark 2. Notice that from all the preceding results, at constant loading amplitudes and periods, plastic accumulation evolves linearly with the number of cycles and can therefore grow indefinitely. However, the materials of engineering interest show that this is not the case at very large numbers of cycles. For instance, one can add isotropic hardening to reach accomodation when a certain plastic equivalent strain is reached. Another way is to sophisticate the model by using non linear hardening rules. This latter is partly what is done in the next application to model the behavior of bituminous materials.

\section{A model adapted to bituminous materials}

In this section, a more elaborated model is proposed within the preceding framework. Having in mind the behavior of bituminous materials, experimental 


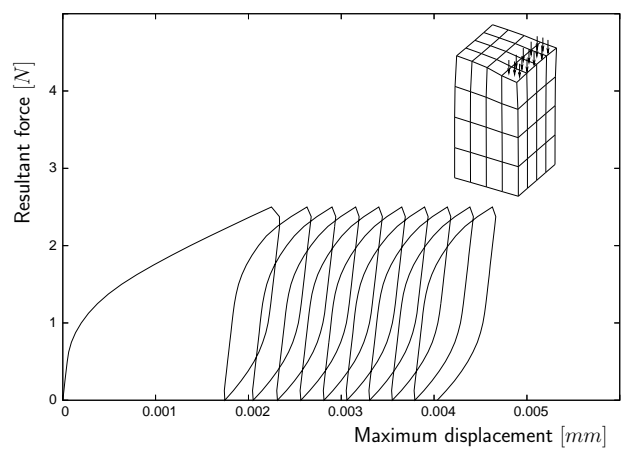

Fig. 4 Non-homogeneous cyclic loading of amplitude $400 \mathrm{KPa}$ and period $\mathrm{T}=40 \mathrm{~s}$.

observations have shown that these latters are compressible and the permanent deformations are usually caused by volumetric densification, specifically for insufficiently compacted materials, followed by shearing at constant volume, see for example $[1,3]$. Hence, a two-mechanisms model is proposed via two distinct kinematic stress-like internal variables: a purely deviatoric tensor $\boldsymbol{X}_{1}$ for shearing and a scalar $X_{2}$ for the volumetric plasticity.

We postulate the following yield criterion which takes the form of a Camclay-like model adapted here for our purposes:

$$
f=\sqrt{\left\|s-\boldsymbol{X}_{1}\right\|^{2}+\frac{2}{3} \varkappa^{2}\left(p-X_{2}+\delta R_{0}\right)^{2}}-\sqrt{\frac{2}{3}} R_{0}
$$

This criterion has the form of an elliptic closed surface of which the initial properties are entirely determined by the parameters $R_{0}, \varkappa$ and $\delta . R_{0}$ is the flow stress, $\varkappa$ characterizes the contribution of the confining stresses, and $\delta$ is introduced in order to ensure the dissimmetry of the behavior between tension and compression. For an illustration, Figure 5 shows this elastic domain at the initial state where, in the $(p, q)$ plane drawing, the quantity $q$ is the so-called von Mises equivalent stress.
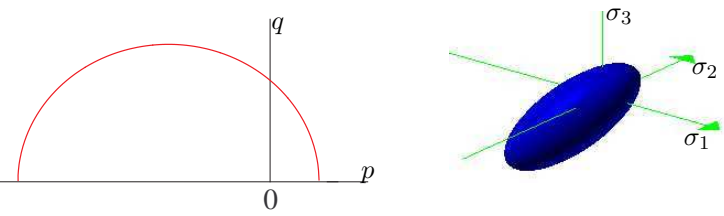

Fig. 5 Initial elastic domain.

Associated plasticity is not suitable for geomaterials as it could not correctly capture the influence of the confining stresses on the volumetric strain 
evolution, see for example $[4,21]$. We postulate then a non-associated plasticity by introducing a flow potential $F$ given by

$$
\begin{aligned}
F & =\sqrt{\left\|s-\boldsymbol{X}_{1}\right\|^{2}+\frac{2}{3} \varpi^{2}\left(p-X_{2}+\delta R_{0}\right)^{2}} \\
& +a\left\|s-\boldsymbol{X}_{1}\right\|+b\left|p-X_{2}\right|
\end{aligned}
$$

where $\varpi$ is a newly introduced parameter for the confining stresse part, and $a$ and $b$ are two parameters that control the nonlinearity of the kinematic hardenings. The associated plasticity could be reached by setting $\varpi=\varkappa$ and $a=b=0$.

Finally, the kinematic hardening restoration is chosen to depend solely on the (deviatoric) back-stress $\boldsymbol{X}_{1}$ via the following potential:

$$
\Omega_{r}=(1-H(f)) \frac{1}{2 \eta_{X}} \boldsymbol{X}_{1}: \boldsymbol{X}_{1}
$$

where $H($.$) is the Heaviside function whose argument is the value of the yield$ function (27). That is, $\Omega_{r}=0$ when $f \geq 0$, and when $f<0, \Omega_{r}$ is positive and the restoration mechanism happens only for pure shear.

The local evolution of the internal variables are then given by the following flow rules of the Perzyna type:

$$
\left\{\begin{array}{l}
\dot{\boldsymbol{\varepsilon}}^{\mathrm{p}}=\frac{\langle f\rangle}{\eta} \frac{\partial F}{\partial \boldsymbol{\sigma}} \\
\dot{\boldsymbol{X}}_{1}=H_{1} \dot{\boldsymbol{e}}^{\mathrm{p}}-H_{1} \frac{\partial \Omega_{r}}{\partial \boldsymbol{X}_{1}} \\
\dot{X}_{2}=\frac{H_{2}}{3} \operatorname{tr}\left[\dot{\boldsymbol{\varepsilon}}^{\mathrm{p}}\right]
\end{array}\right.
$$

where use has been made of the decomposition $\varepsilon^{\mathrm{p}}=\boldsymbol{e}^{\mathrm{p}}+\operatorname{tr}\left[\varepsilon^{\mathrm{p}}\right] \mathbf{1} . H_{1}$ and $H_{2}$ are the kinematic hardening moduli for the deviatoric and volumetric parts, respectively.

- For $f>0$, we have $\Omega_{r}=0$ from Equation (29), and the precedent evolution equations has a classical form.

- For $f \leq 0$, the local evolution equations (30) reduce to

$$
\dot{\varepsilon}^{\mathrm{p}}=\mathbf{0}, \quad \dot{\boldsymbol{X}}_{1}+\frac{H_{1}}{\eta_{X}} \boldsymbol{X}_{1}=\mathbf{0}, \quad \dot{X}_{2}=0
$$

For the sake of clarity, details about the numerical discretization are here omitted for this model, see for this [9]. Its finite element implementation follows the main steps adopted for the model example of Section 4. 
5.1 A numerical parametric study

In order to demonstrate the performance of the present model and to justify the choices for the material parameters, a set of simple numerical examples are presented below. For this, we consider a cylindrical sample under triaxial loading with lateral confining pressure:

$$
\boldsymbol{\sigma}=\left[\begin{array}{lll}
\sigma_{\mathrm{a}} & & \\
& \sigma_{\mathrm{c}} & \\
& & \sigma_{\mathrm{c}}
\end{array}\right]
$$

where $\sigma_{\mathrm{a}}$ and $\sigma_{\mathrm{c}}$ are respectively the axial and the confining stresses. The volumetric plastic strain $\varepsilon_{\mathrm{v}}^{\mathrm{p}}$ and the shear plastic strain $\varepsilon_{s}^{\mathrm{p}}$ are then computed as follows:

$$
\varepsilon^{\mathrm{p}}=\left[\begin{array}{lll}
\varepsilon_{\mathrm{a}}^{\mathrm{p}} & & \\
& \varepsilon_{\mathrm{c}}^{\mathrm{p}} & \\
& & \varepsilon_{\mathrm{c}}^{\mathrm{p}}
\end{array}\right], \quad \varepsilon_{\mathrm{v}}^{\mathrm{p}}=\operatorname{tr}\left[\varepsilon^{\mathrm{p}}\right], \quad \varepsilon_{\mathrm{s}}^{\mathrm{p}}=\frac{2}{3}\left(\varepsilon_{\mathrm{a}}^{\mathrm{p}}-\varepsilon_{\mathrm{c}}^{\mathrm{p}}\right)
$$

The material parameters are chosen as follows: the Young's modulus $E=$ $3000 \mathrm{MPa}$, the Poisson ratio $\nu=0.35$, and for the yield criterion $(30), R_{0}=$ $0.1 M P a, \varkappa=0.9$ and $\delta=0.75$.

Both static and cyclic creep tests are considered. The volumetric and shear plastic strains as functions of the loading durations (time or number of loading cycles) are studied. For the static creep, the applied stresses are $\sigma_{a}=0.267 \mathrm{MPa}$ and $\sigma_{c}=0.167 \mathrm{MPa}$. And for the cyclic creep, the precedent values are the amplitudes at a frequency of $1 \mathrm{~Hz}$ (that is, the mean stresses are $\bar{\sigma}_{a}=0.133 \mathrm{MPa}$ and $\bar{\sigma}_{c}=0.083 \mathrm{MPa}$ ).

Figure 6 shows the responses for two particular cases of kinematic hardening: case 1 with $H_{1}=0$ and $H_{2}=80 \mathrm{MPa}$, and case 2 with $H_{1}=65 \mathrm{MPa}$ and $H_{2}=0$. In both cases, the parameters appearing in the plastic potential $F$ in Equation (28) are $\varpi=1.15, a=b=0$, the viscous parameters are $\eta=0$ and $\eta_{X}=2.6510^{2}$ MPas. We observe that when activating only one kinematic hardening, the model is unable to maintain simultaneously the evolution of volumetric and shear strains under static as well as under cyclic creep loadings. The introduction of both of the two kinematic hardening mechanisms is then necessary for the modeling.

On the other hand, fixing the values of the kinematic hardening moduli $H_{1}$ and $H_{2}$ (here with $H_{1}=65 \mathrm{MPa}$ and $\mathrm{H}_{2}=80 \mathrm{MPa}$ ), the nonlinearities of both of the kinematic hardenings are required. This is proved by the responses of Figure 7 for particular cases of the parameters $a$ and $b$, i.e. the two parameters controlling the nonlinear evolutions of $\boldsymbol{X}_{1}$ and $X_{2}$, respectively (again see Equation (28)).

One can observe as well from Figure 7 that these nonlinearities induce a linear evolution of the permanent strain versus the loading duration. But this evolution trend is not conform to the experimental observations in general. It is then necessary to introduce one or several parameters which evolve with the loading history. As the permanent strain rates are inversely proportional to the 

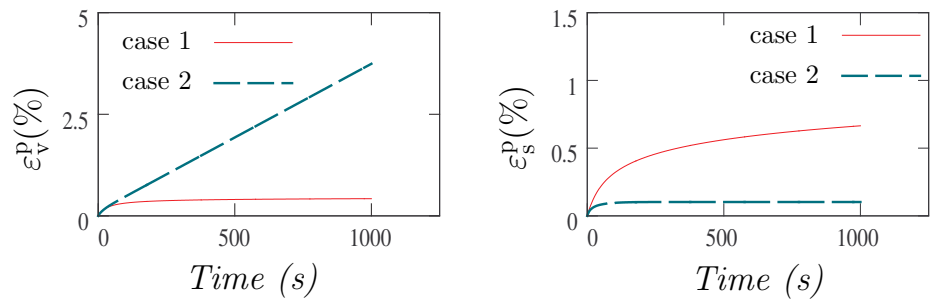

$a$ - Static creep tests

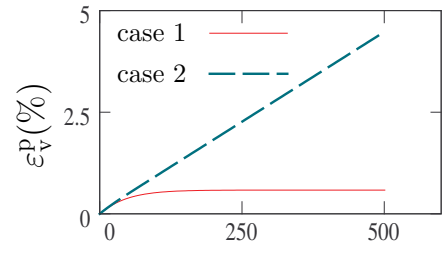

Cycles number

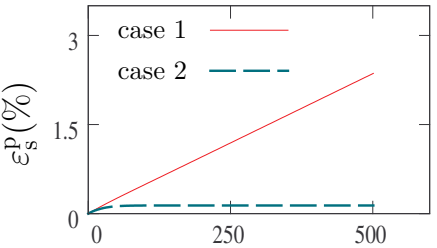

Cycles number

$b$ - Cyclic creep tests

Fig. 6 Responses of the model for two particular cases of $H_{1}$ and $H_{2}$ (case 1: with $H_{1}=0$ and $H_{2}=80 \mathrm{MPa}$, and case 2: with $H_{1}=65 \mathrm{MPa}$ and $\left.H_{2}=0\right)$.
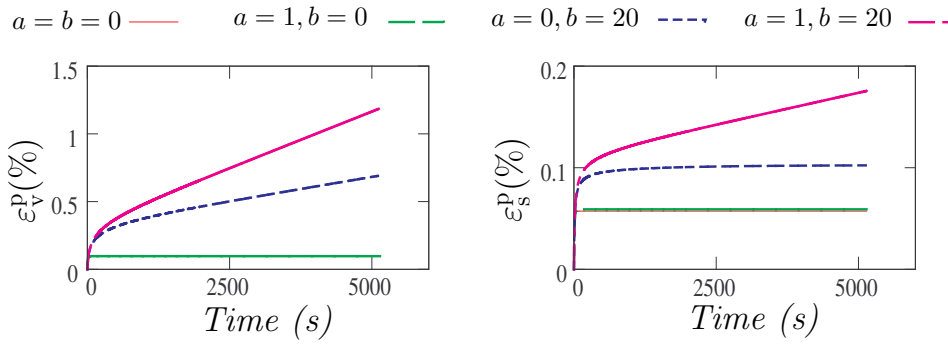

$a$ - Static creep tests

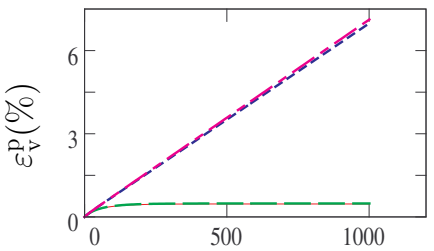

Cycles number

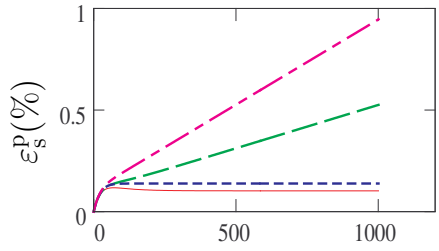

Cycles number

$b$ - Cyclic creep tests

Fig. 7 Responses of the model for particular cases of the parameters $a$ and $b$. 
viscous parameter $\eta, \eta$ is thus chosen to depend on the cumulative permanent strain $\bar{e}^{\mathrm{p}}$ which is widely used to characterize the plastic strain history, see for example $[17,18]$ :

$$
\eta=\eta_{0}\left(1+\eta_{1} \bar{e}^{\mathrm{p}} \exp \left[\eta_{2} \bar{e}^{\mathrm{p}}\right]\right), \quad \bar{e}^{\mathrm{p}}(t)=\int_{\tau=0}^{t} \sqrt{\frac{3}{2} \dot{\varepsilon}^{\mathrm{p}}: \dot{\boldsymbol{\varepsilon}}^{\mathrm{p}}} d \tau
$$

where $\eta_{0}$ is the initial viscous parameter, $\eta_{1}$ and $\eta_{2}$ are positive parameters. Along with loading duration, $\bar{e}^{\mathrm{p}}$ increases, $\eta$ increases too leading then to the decrease of the permanent strain rates. Likewise, to control the evolution of cyclic permanent strains, we also postulate a progressive change for the restoration parameter $\eta_{X}$ as follows:

$$
\eta_{X}=\eta_{X_{0}}\left(1+\eta_{X_{1}}\left(\exp \left[\eta_{X_{2}} \bar{e}^{\mathrm{p}}\right]-1\right)\right)
$$

where $\eta_{X_{0}}, \eta_{X_{1}}$ and $\eta_{X_{2}}$ are positive parameters. We use these new enhancements in the next modeling example.

\subsection{Parameter identification}

Of concern in this section, some indications of how the various material parameters can be identified. By first noticing that the three parameters $R_{0}, \varkappa$ and $\delta$ characterize the elastic domain, see Figure 5, they can then be determined in a usual manner from elastic limits for different loading paths.

Now by considering the model response from the precedent parametric study and its further extensions given by the constitutive relations (34) and (35), one finds that the $\varepsilon_{\mathrm{v}}^{\mathrm{p}}(t)$ and $\varepsilon_{\mathrm{s}}^{\mathrm{p}}(t)$ curves for the static creep and the $\varepsilon_{\mathrm{v}}^{\mathrm{p}}(N)$ and $\varepsilon_{\mathrm{s}}^{\mathrm{p}}(N)$ curves for the cyclic creep can be divided into two parts. The first part, that we denote by Phase 1, corresponds to the early response where the permanent strains increase quickly. The next part, that we denote by Phase 2, consists to a somehow stabilized phase where the permanent strains increase slowly.

Parameters $H_{1}, H_{2}, a, b, \eta_{1}$ and $\eta_{2}$ mostly influence the whole response in static creep. In particular, $H_{1}, a, H_{2}$ and $b$ strongly influence the Phase 1 responses, while Phase 2 is mostly influenced by $\eta_{1}$ and $\eta_{2}$. On the other hand, for cyclic creep, the volumetric response is mostly influenced by the parameters $\varpi$ and $\eta_{0}$ in Phase 1, and by the parameter $\eta_{2}$ in Phase 2.

At last, as the constitutive relation (35) solely influences the cyclic creep responses, mostly for the shear response, the paramaters $\eta_{X_{0}}, \eta_{X_{1}}$ and $\eta_{X_{2}}$ can be determinined from the $\varepsilon_{\mathbf{s}}^{\mathrm{p}}(N)$ curves.

With these observations at hand, we propose the following identification procedure by curve fitting of different permament evolution responses:

- Parameters $\varpi$ and $\eta_{0}$ are determined from Phase 1 of static and cyclic creep tests. 
- Paramaters $H_{1}, a, H_{2}$ and $b$ are determined by curve fitting $\varepsilon_{\mathrm{v}}^{\mathrm{p}}(t)$ and $\varepsilon_{\mathrm{s}}^{\mathrm{p}}(t)$ under static creep.

- Parameter $\eta_{1}$ is determined from Phase 1 of the volumetric response $\varepsilon_{\mathrm{v}}^{\mathrm{p}}(N)$ under cyclic creep.

- Parameter $\eta_{2}$ is determined from Phase 2 responses of the $\varepsilon_{\mathrm{v}}^{\mathrm{p}}(t), \varepsilon_{\mathrm{s}}^{\mathrm{p}}(t)$ and $\varepsilon_{\mathrm{v}}^{\mathrm{p}}(N)$ curves.

- Parameters $\eta_{X_{0}}, \eta_{X_{1}}$ and $\eta_{X_{2}}$ are determined by curve fitting of the whole cyclic creep response $\varepsilon_{\mathrm{s}}^{\mathrm{p}}(N)$.

These identifications need then triaxial experimental tests under static and cyclic creep, preferably at loading levels of the same order.

\subsection{A comparison with selected experimental data}

In this section, some simulations of creep tests are compared to some experimental results by [1]. These authors have in fact realized a complete test campaign to study permanent behaviors of asphalt mixtures. All the tests were processed on cylindrical specimens of length $240 \mathrm{~mm}$. Both static and cyclic creep tests were performed under triaxial loadings with the presence of lateral confining stresses in order to limit the instability of the samples. In cyclic creep tests, the square waves stress form was involved with the stress on for $0.5 s$ and off for $0.5 s$. The stress levels in the corresponding static creep tests were equal to the peak values in the cyclic creep tests. Axial and radial permanent strains were measured and used to calculate the volumetric and shear strains after Equations (33).

The static and cyclic creep tests at stress levels of $\sigma_{\mathrm{a}}=0.267 \mathrm{MPa}$ and $\sigma_{\mathrm{c}}=0.167 \mathrm{MPa}$ were chosen to validate the proposed model. The Young's modulus and the Poisson ratio of the materials were [1]: $E=830 \mathrm{MPa}$ and $\nu=0.25$. Within the present framework, the following set of parameters has been identified, see [9] for details:

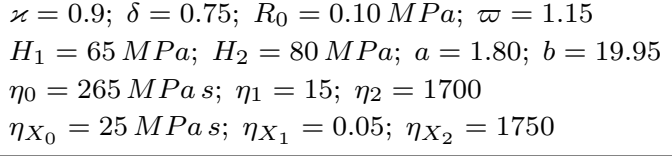

With these material parameters, Figure 8 shows the numerical responses of the model under static creep loading. Thanks to the presence of two different kinematic hardening mechanisms, both the volumetric and shear evolutions are in satisfactory good agreement with the experimental results. The non-linearity of the kinematic hardening stresses allow the strains rates to be maintained until a high loading duration. The evolution of the viscous parameter $\eta$ brings a diminution of permanent strains rates, and thus a nonlinear evolution of $\varepsilon_{\mathrm{v}}^{\mathrm{p}}$ and $\varepsilon_{\mathrm{s}}^{\mathrm{p}}$ versus time. 

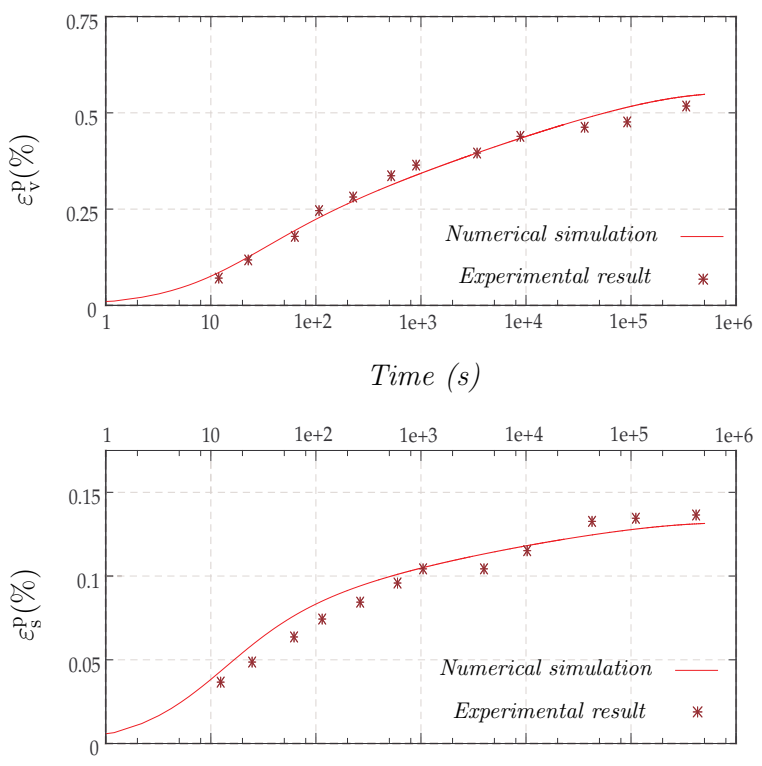

Fig. 8 Static creep test. Comparison with experimental results of Brown and Cooper [1].

Also, a comparison of the numerical responses and the experimental results in the case of cyclic creep tests is presented in Figure 9. Here again, a good correlation confirms the capacities of the proposed model. The restoration of shear kinematic hardening stress $\boldsymbol{X}_{1}$ ensures a higher permanent strain level in cyclic creep tests than in static creep tests.

\section{Conclusion and perspectives}

In this paper we have presented a viscoplastic constitutive framework suitable for the prediction of the cyclic loadings at high number of cycles. The model is based on the notion of kinematic hardening restoration that is physically observed in many man-made materials of engineering interest.

Motivated by a simple rheological model with elementary considerations, we have shown that the three-dimensional constitutive modeling is straightforward and in agreement with the continuum thermodynamics requirements. We have shown that using a single-surface yield criterion, one can get plastic accumulation at a very large number of cycles.

An application to describe the behavior of bituminous materials has been proposed. We have shown that this latter is richly featured and is capable of simulating not only cyclic creep loadings, but also static creep loadings at the same time. However, as bituminous materials are very complex materials in general, the proposed model should be improved to account for many other 


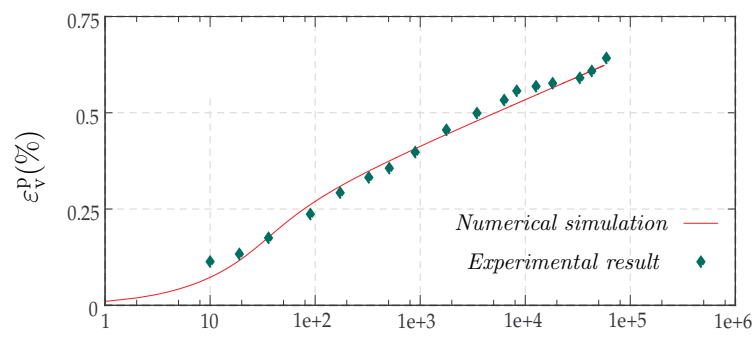

Number of loading cycles

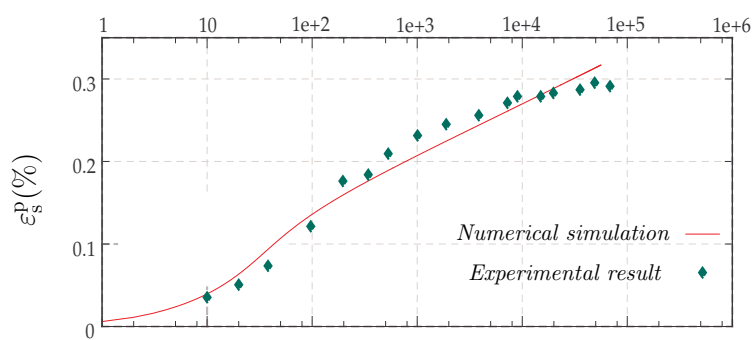

Fig. 9 Cyclic creep test. Comparison with the experimantal results of Brown and Cooper [1].

characteristics such as the omnipresent temperature dependency in the asphalt concrete pavements, and damage in terms of crack and air void growth.

\section{References}

1. Brown, S.F., Cooper, K.E.: A fundamental study of the stress-strain characteristics of a bituminous materials. Journal of the Association of Asphalt Paving Technologists 49, 476-497 (1980)

2. Cailletaud, G., Saï, K.: Study of plastic/viscoplastic models with various inelastic mechanisms. International Journal of Plasticity 11(8), 991-1005 (1995)

3. Eisenmann, J., Hilmer, A.: Influence of wheel load and inflation pressure on the rutting effect at asphalt-pavements - experiments and theoretical investigations. In: 6th International Conference on the Structural Design of Asphalt Pavements, vol. 1, pp. 392-403. Ann Arbor (1987)

4. Florea, D.: Nonassociated elastic/viscoplastic model for bituminous concrete. Int. Journal of Engineering Science 32, 87-93 (1994)

5. Germain, P., Nguyen, Q., Suquet, P.: Continuum thermodynamics. ASME Journal of Applied Mechanics 50, 1010-1021 (1983)

6. Khoei, A.R., Jamali, N.: On the implementation of multi-surface kinematic hardening plasticity and its applications. International Journal of Plasticity 21, 1741-1770 (2005)

7. Lemaitre, J., Chaboche, J.L.: Mechanics of Solid Materials. Cambridge University Press, London (1990)

8. Mandel, J.: Généralisation de la théorie de w.t. koiter. International Journal of Solids and Structures 1, 273-295 (1965)

9. Nguyen, D.T.: Prédiction des déformations irréversibles des couches de surface des chaussées bitumineuses (in french). Ph.D. thesis, Ecole Nationale des Ponts et Chaussées, Paris (2006) 
10. Nguyen, D.T., Nedjar, B., Tamagny, P.: Cyclic elasto-viscoplastic model for asphalt concrete materials. International Journal for Road Materials and Pavement Design 8(2), 239-255 (2007)

11. Ohno, N.: Current state of the art in constitutive modeling for ratcheting behavior. In: Proceedings of the 14th International Conference on SMiRT, pp. 201-212. Lyon (1997)

12. Ohno, N., Wang, J.D.: Kinematic hardening rules with critical state of dynamic recovery, part I: formulations and basic features for ratcheting behavior. International Journal of Plasticity 9, 375-390 (1993)

13. Perzyna, P.: Fundamental problems in viscoplasticity. Advances in Applied Mechanics 9, 243-377 (1966)

14. Scarpas, A., Al-Khoury, R., Van Gurp, C., Erkens, S.M.: Finite element simulation of damage development in asphalt concrete pavements. In: Proceedings of 8 th International Conference on Asphalt Pavements, pp. 673-692. University of Washington, Seattle, WA (1997)

15. Seibi, A.C., Sharma, M.G., Ali, G.A., Kenis, W.J.: Constitutive relations for asphalt concrete under high rates of loading. In: Transportation Research Record 1767, Transportation Research Board, National Research Council, pp. 111-119. Washington, D.C. (2001)

16. Sides, A., Uzan, J., Perl, M.: A comprehensive visco-elastoplastic characterization of sand-asphalt under compression and tension cyclic loading. ASTM Journal of Testing and Evaluation 13, 49-59 (1985)

17. Simo, J., Hughes, T.: Computational Inelasticity. Springer-Verlag, New York (1998)

18. Simo, J.C.: Numerical analysis and simulation of plasticity. In: P. Ciarlet, J. Lions (eds.) Handbook of Numerical Analysis, vol. VI, pp. 183-499. North-Holland (1998)

19. Tashman, L., Masad, E., Little, D., Zbib, H.: A microstructure-based viscoplastic model for asphalt concrete. International Journal of Plasticity 21, 1659-1685 (2005)

20. Yoshida, F.: A constitutive model of cyclic plasticity. International Journal of Plasticity 16, 359-380 (2000)

21. Zienkiewicz, O., Humpheson, C., Lewis, R.: Associated and non-associated viscoplasticity in soils mechanics. Journal of Geotechnique 25(5), 671-689 (1975) 